\title{
Accurate Position Sensing of Defocused Beams Using Simulated Beam Templates
}

A. Awwal, J. Candy, C. Haynam, C. Widmayer, E. Bliss, S. Burkhart

September 30, 2004

SPIE Annual Meeting 2004

Denver, CO, United States

August 2, 2004 through August 5, 2004 
This document was prepared as an account of work sponsored by an agency of the United States Government. Neither the United States Government nor the University of California nor any of their employees, makes any warranty, express or implied, or assumes any legal liability or responsibility for the accuracy, completeness, or usefulness of any information, apparatus, product, or process disclosed, or represents that its use would not infringe privately owned rights. Reference herein to any specific commercial product, process, or service by trade name, trademark, manufacturer, or otherwise, does not necessarily constitute or imply its endorsement, recommendation, or favoring by the United States Government or the University of California. The views and opinions of authors expressed herein do not necessarily state or reflect those of the United States Government or the University of California, and shall not be used for advertising or product endorsement purposes. 


\title{
Accurate position sensing of defocused beams using simulated beam templates
}

\author{
Abdul Awwal, James Candy, Chris Haynam, Clay Widmayer, Erlan Bliss, \\ and Scott Burkhart \\ National Ignition Facility \\ Lawrence Livermore National Laboratory, Livermore, CA. 94551 \\ E-mail: awwal1@1lnl.gov
}

\begin{abstract}
In position detection using matched filtering one is faced with the challenge of determining the best position in the presence of distortions such as defocus and diffraction noise. This work evaluates the performance of simulated defocused images as the template against the real defocused beam. It was found that an amplitude modulated phase-only filter is better equipped to deal with real defocused images that suffer from diffraction noise effects resulting in a textured spot intensity pattern. It is shown that the there is a tradeoff of performance dependent upon the type and size of the defocused image. A novel automated system was developed that can automatically select the right template type and size. Results of this automation for real defocused images are presented.
\end{abstract}

Key word: pattern recognition, matched filtering, amplitude modulated phase only filter, AMPOF, phase-only filter, target tracking, optical alignment, automated optical alignment

\section{INTRODUCTION}

The National Ignition Facility (NIF), currently under construction at the Lawrence Livermore National Laboratory, is a stadium-sized facility containing a 192-beam, 1.8-megajoule, 500-terawatt, ultraviolet laser system for the study of inertial confinement fusion and the physics of matter at extreme energy densities and pressures [1]. Automatic alignment is one of the most important operations on NIF. At the heart of this technique is the beam position detection algorithm. Alignment of laser beams requires finding the position of an imaged beam feature at various locations along the beam path. The position of the beam is determined by finding the location of the shadow of a fiducial inserted into the beam. These images of the fiducials are gathered using a fixed-focus CCD camera. In different beams the fiducials are out of focus by varying amounts. Compounded with this are the diffraction spots arising from dust on the optics along with a change in the illumination gradient in the optical system.

Traditional approaches to finding the position of such beams are based on centroiding. In this approach, the image is subjected to various filtering and thresholding, resulting in a segmentation of the desired spot from the background; centroiding is then performed on the segmented spot. However, due to defocus and diffraction noises, the shape of this segmented image is seldom circular. Thus the centroid location is heavily biased by the presence of the diffraction noise, illumination gradient, and defocus.

\section{THE PROBLEM}

Four examples of beam images are depicted in Fig. 1. The beams show the shadow of the fiducial located in the center of the beam. The algorithm needs to determine the position of these beams by finding the position of the dark spot at the center. However it should be noted that although the focused spot is supposed to be circular, noise and other diffraction effects resulting from dust spots in the optics make it difficult to apply a simple centroid-based technique. Due to the variation of intensity inside the dark region it is hard to obtain a circular region by simple thresholding. Looking at a one- 
dimensional slice may not reveal the complexity of the problem. In any one specific direction a simple threshold may convert the distorted top hat into a clean circular cross-section, but in the other direction the same threshold may lose half of the beam. Thus a one-dimensional analysis of two-dimensional problems generally hides the complexity of this kind of problem. In this paper we explore the possibility of utilizing template-based matched filtering for detecting the beam positions (Fig. 1). The idea is to use simulated beam images experiencing the same amount of defocus as the template for the real beam with the same defocus. The background for the matched filter used is described in the next section.
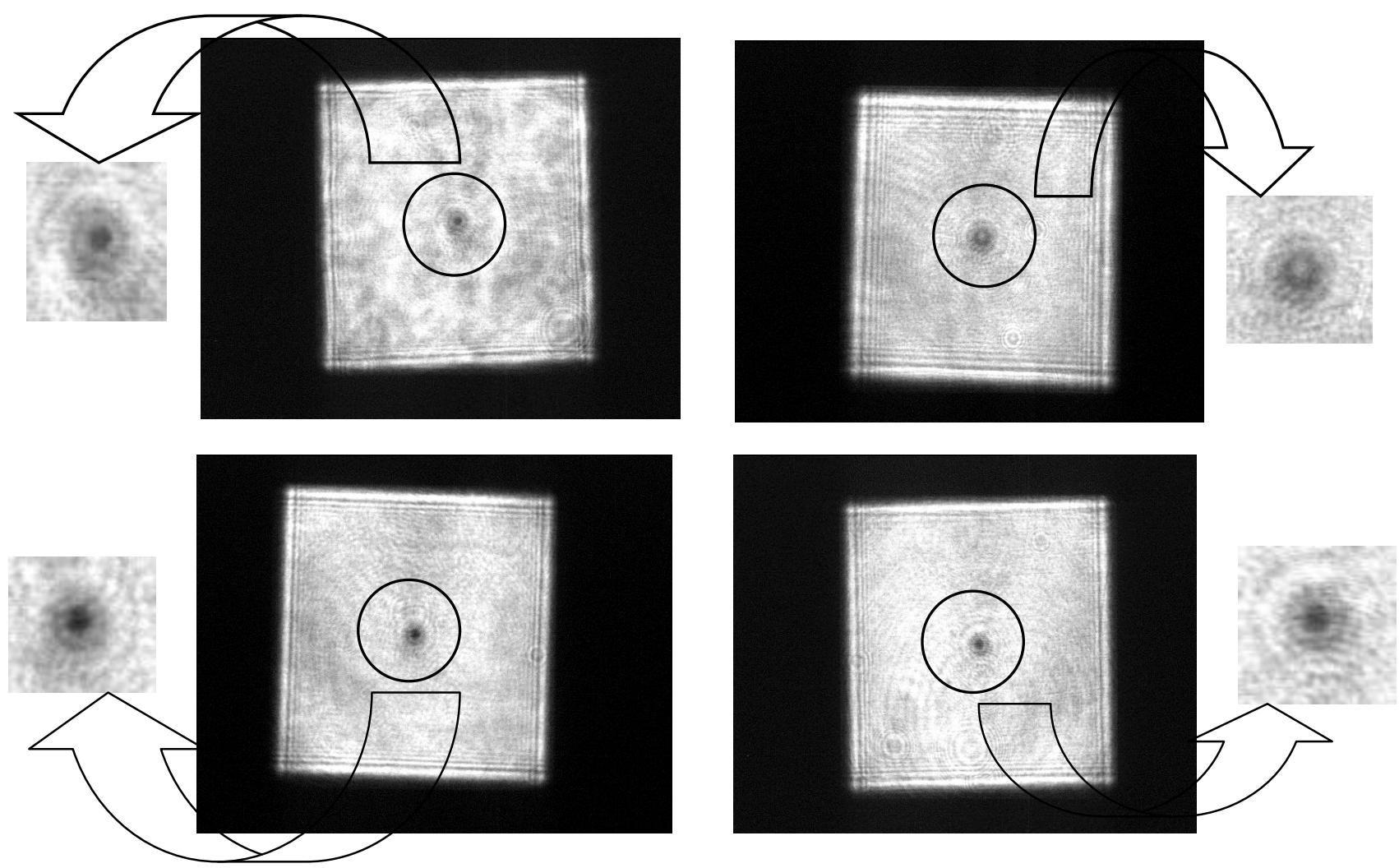

Fig. 1: Examples of real defocused beam fiducial

\section{BACKGROUND}

VanderLugt introduced the classical matched filter for optical pattern recognition; wherein the complex amplitude and phase of the reference pattern is stored as a hologram [2]. The phase-only filter (POF) is a variation of the complex match filter (CMF) that uses only the phase of the reference pattern to perform correlation detection [3]. The amplitude modulated phase-only filter (AMPOF) was designed to further enhance the performance of the POF [4]. The mathematical foundation of the AMPOF filter is derived as follows:

Let the Fourier transform of the object function $f(\mathrm{x}, \mathrm{y})$ be denoted by:

$$
F\left(U_{x}, U_{y}\right)=\left|F\left(U_{x}, U_{y}\right)\right| \exp \left(j \Phi\left(U_{x}, U_{y}\right)\right)
$$


A CMF corresponding to this function $f(\mathrm{x}, \mathrm{y})$ is expected to produce its autocorrelation. From the Fourier transform theory of correlation the CMF is given by the complex conjugate of the input Fourier spectrum as denoted by Eq. 2:

$$
H_{C M F}\left(U_{x}, U_{y}\right)=F^{*}\left(U_{x}, U_{y}\right)=\left|F\left(U_{x}, U_{y}\right)\right| \exp \left(-j \Phi\left(U_{x}, U_{y}\right)\right)
$$

The inverse Fourier transformation of the product of $F\left(\mathrm{U}_{\mathrm{x}}, \mathrm{U}_{\mathrm{y}}\right)$ and $H_{C M F}\left(\mathrm{U}_{\mathrm{x}}, \mathrm{U}_{\mathrm{y}}\right)$ results in the convolution of $f(\mathrm{x}, \mathrm{y})$ and $f(-\mathrm{x},-\mathrm{y})$, which is the equivalent of the autocorrelation of $f(\mathrm{x}, \mathrm{y})$. Moreover, when $\left|F\left(\mathrm{U}_{\mathrm{x}}, \mathrm{U}_{\mathrm{y}}\right)\right|$ is set to unity, $H_{C M F}$ becomes a phase-only filter (POF):

$$
H_{P O F}\left(U_{x}, U_{y}\right)=\exp \left(-j \Phi\left(U_{x}, U_{y}\right)\right)
$$

The phase only correlation of the input image and the target is simply:

$$
C_{P O F}(\Delta \mathrm{x}, \Delta \mathrm{y})=F^{-1}\left\{F\left(\mathrm{U}_{\mathrm{x}}, \mathrm{U}_{\mathrm{y}}\right) H_{P O F}\left(\mathrm{U}_{\mathrm{x}}, \mathrm{U}_{\mathrm{y}}\right)\right\}
$$

Since the convolution operator in the spatial domain is equivalent to the product operator in the frequency domain, one can think of the POF as an edge enhancer by way of division by $\left|F\left(\mathrm{U}_{\mathrm{x}}, \mathrm{U}_{\mathrm{y}}\right)\right|$. A more generalized treatment $\left[{ }_{5,6}\right]$ can be given as follows, where the generalized AMPOF filter is expressed as:

$$
H_{C M F}\left(U_{x}, U_{y}\right)=\frac{a F^{*}\left(U_{x}, U_{y}\right)}{\left[b+c\left|F\left(U_{x}, U_{y}\right)\right|+d\left|F\left(U_{x}, U_{y}\right)\right|^{2}\right]^{m}}
$$

When $\mathrm{a}=\mathrm{b}=\mathrm{m}=1$ and $\mathrm{c}=\mathrm{d}=0$, this results in the classical matched filter; when $\mathrm{b}=\mathrm{d}=0$ and $\mathrm{a}=\mathrm{c}, \mathrm{m}=1$ it results in the phase only filter expressed by Eq. 3. When $b$ is a small constant for nonzero values of a, c, and d, it is an AMPOF. The AMPOF described in Ref. 4 has $\mathrm{a}=$ constant, $\mathrm{d}=$ constant, $\mathrm{c}=0, \mathrm{~m}=1$ and $\mathrm{b}=\varepsilon$ (a small constant number). It was found after some experimentation that when $\mathrm{b}=\varepsilon, \mathrm{d}=0$, and $\mathrm{m}=\mathrm{c}=\mathrm{a}=1$, it resulted in lower variance in the beam positions. The position of the object can be found from the position of the crosscorrelation, autocorrelation, and the position of the template using the Eqs. 6-7.

$$
\begin{aligned}
& \mathrm{x}_{\text {pos }}=\mathrm{x}_{\text {cross }}-\mathrm{x}_{\text {auto }}+\mathrm{x}_{\mathrm{c}} \\
& \mathrm{y}_{\text {pos }}=\mathrm{y}_{\text {cross }}-\mathrm{y}_{\text {auto }}+\mathrm{y}_{\mathrm{c}}
\end{aligned}
$$

Where $\left(\mathrm{x}_{\mathrm{pos},}, \mathrm{y}_{\mathrm{pos}}\right)$ is the to-be-determined position of the pattern in the image plane, $\left(\mathrm{x}_{\text {auto }}, \mathrm{y}_{\text {auto }}\right)$ is the position of the tempate autocorrelation peaks and ( $\left.\mathrm{x}_{\text {cross }}, \mathrm{y}_{\text {cross }}\right)$ is the position of the cross correlation peak. The position of the crosscorrelation peak was estimated using a polynomial fit of second order to the correlation peak. The center of the template,

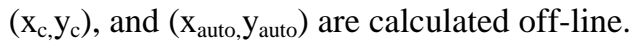




\section{ALGORITHM}

Our proposed approach is to use a simulated defocused beam as a template with the same amount of defocus as the real beam. This is feasible in cases where there is only a single beam or fixed amount of defocus. However, if the same algorithm must process all the beams when multiple beams have different amounts of defocus, then one must be able to select the right template. One of the most important questions that must be answered is how do we select the right filter for a real beam? The second important question is what the size of the template for a given image should be. A more fundamental question may be does the size of the template matter? In order to answer these questions we examine the computer simulation of a circular spot through different defocusing (Fig. 2). The varying amounts of defocus result from changing the laser beam path length to delay the arrival of the laser pulse to the target chamber $\left.{ }_{1}\right]$. Therefore the defocus is expressed as a time delay. There are two distinct characteristics, in one case it results in a smaller dark inner spot (Fig. 2, right image), in the second it results in an enlarged spot (Fig. 3, right image). In both cases there may also be a different gradient present.
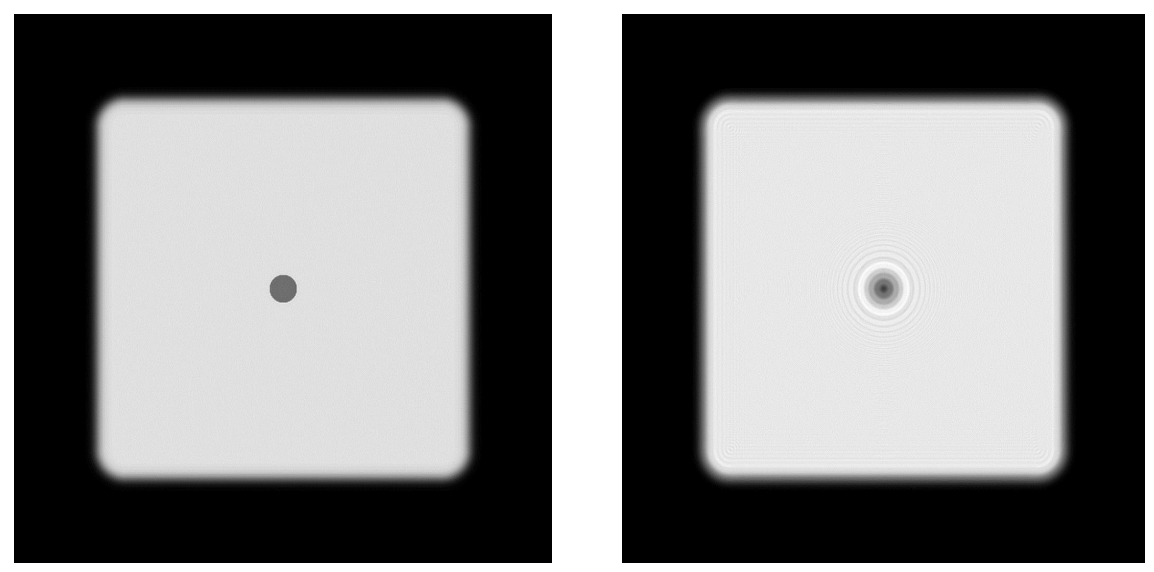

Fig. 2: Simulated images with no noise with 0ns and +6ns delay
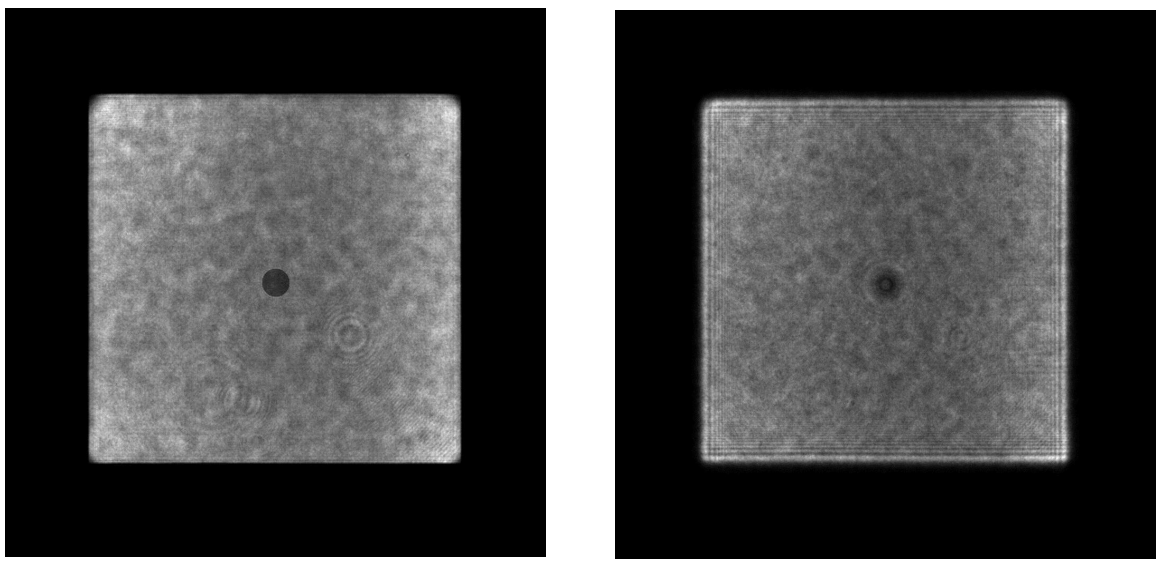

Fig. 3: Simulated images with noise with 0ns and 3ns delay

These characteristics can also be observed from the cross-section of the simulated beam templates shown in Figs. 4 and 5 . Note that $\pm 3 \mathrm{~ns}$ delay results in an expanded center spot. The $6 \mathrm{~ns}$ to $13 \mathrm{~ns}$ beam delays result in a smaller spot with a graded intensity pattern (Fig. 4). The real beams, corresponding to two types of beam delays applied to four separate beams, are shown in Fig. 5. Note there are two distinct types of defocus. One of the beams exhibits an expanded center spot, while the other three show tight dark spots with varying intensity gradients. 


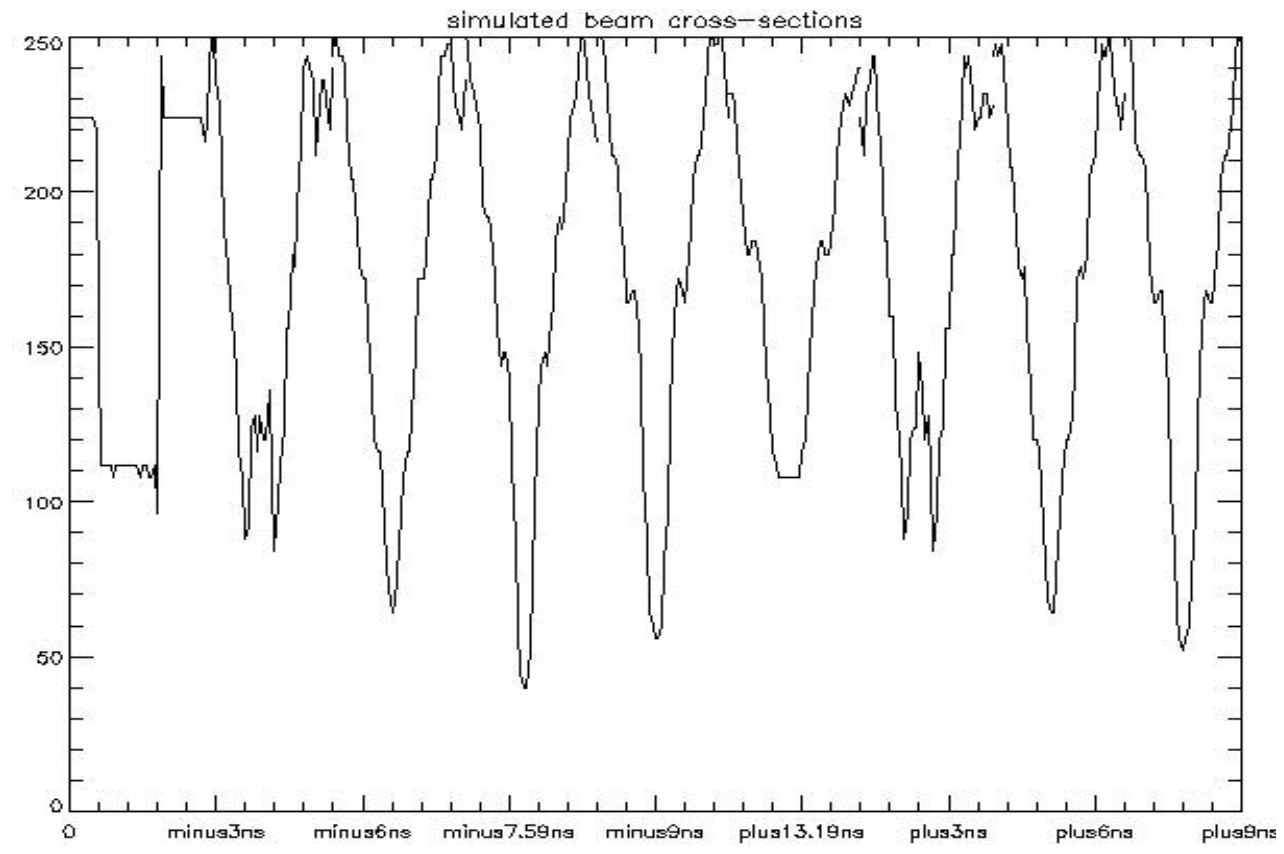

Fig. 4: The cross-section of the simulated defocused center spot for 9 different beam delays

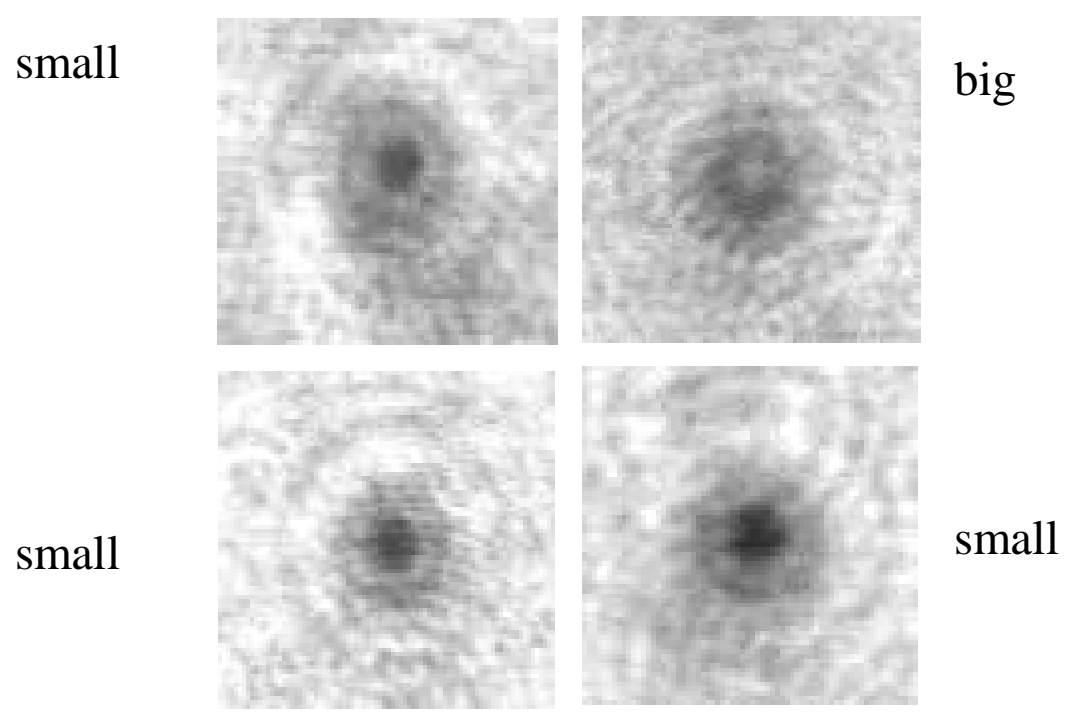

Fig. 5: The cross-section of the 4 real defocused center spots for 2 different beam delays 


\section{EXPERIMENTS}

First we use a simulated focused beam as the template and then apply the template to all the simulated defocused images with a noiseless and noisy background. There are a total of 36 images with 9 different degrees of defocus. The scatter plot from this experiment is shown in Fig. 6. The 3-sigma standard deviation (3-sigma) for this experiment is less than 0.1 pixel for all simulated defocus cases. This is a surprising result illustrating that when simulated, the defocused center spots maintain their circular symmetry.

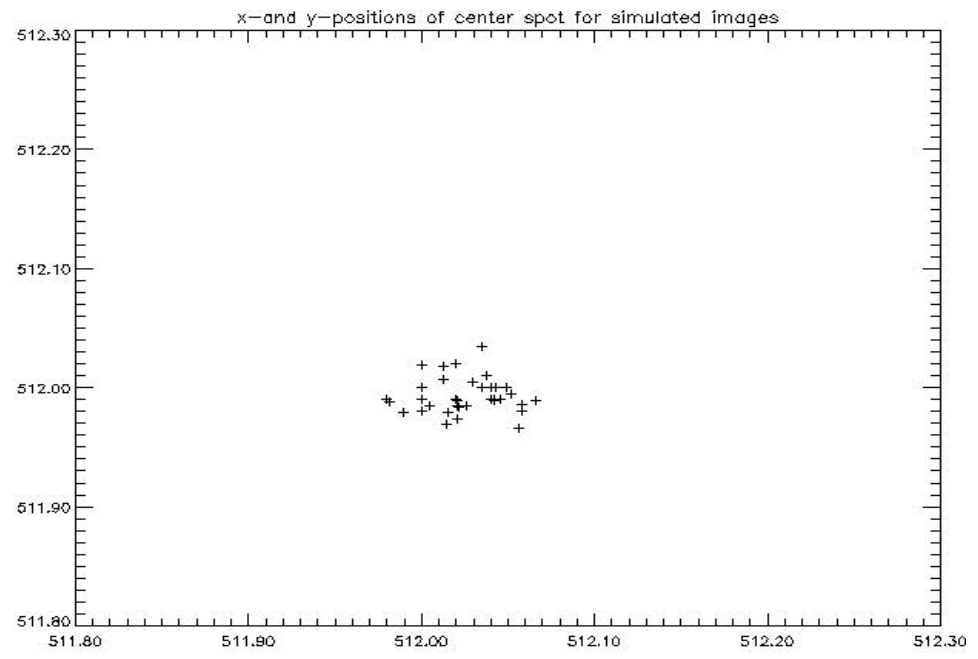

Fig. 6: The scatter plot of position estimates from a set of 36 simulated beams against a simulated beam template

Next we apply a simulated beam template to detect beam 318, which has a small defocused center spot. It should be noted that the small spot (Fig. 7a), visually matches the $6 \mathrm{~ns}$ to $13.19 \mathrm{~ns}$ beam images. However, we chose $13.19 \mathrm{~ns}$ as the template, since it resembles the $7.72 \mathrm{~ns}$ delayed real beam. In order to evaluate the performance, a set of real beam images collected from 30 different successive instances is used. Ignoring vibrations due to the opto-mechanical components, the spot should be detected at the same position for every case. It was found that with the template truncated to a 14-pixel radius the 3 -sigma was about 0.42 pixels where sigma is the standard deviation of the 30 detected image positions.
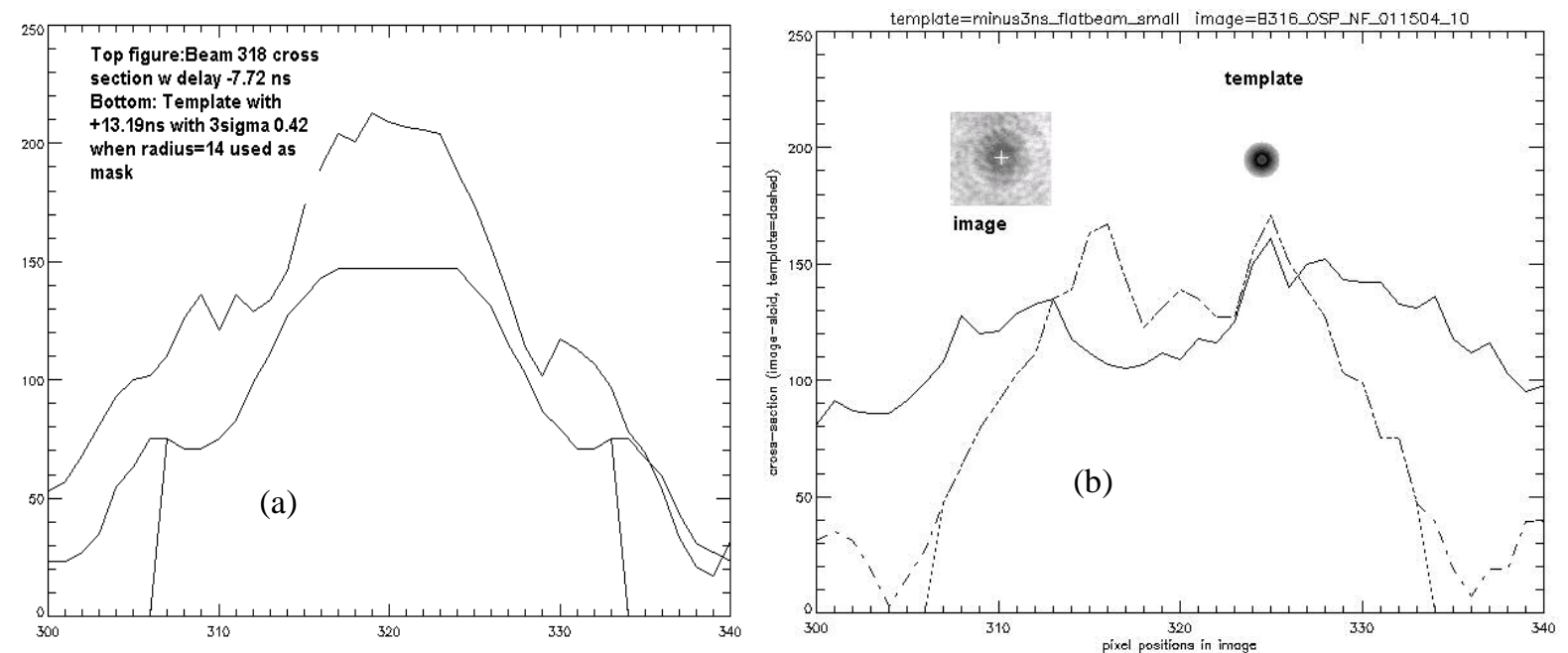

Fig. 7: The cross-section of the real and simulated defocused beam 
Next we apply the technique to the expanded center spot shown in Fig. 7b. It was found that this particular beam, with a relatively bright inner core, resembles the 3ns-delayed beam. The cross-section is shown in Fig. $7 \mathrm{~b}$ where the solid lines represent the real beam and the dashed line represents the template.

This particular beam was tested with two different template sizes (radius of 12 and 14). With radius 12 the 3 -sigma was found to be 0.36 pixels. The same template with a 10-or 14-pixel radius produced a 3-sigma of more than a pixel. A comparison of CMF and AMPOF was made; the AMPOF showed better results since it is less sensitive to the absolute intensity. The CMF is biased toward the higher beam intensity while AMPOF is more sensitive to the edges, as evidenced from the inset of Fig. 8a-b where the cross hair is biased to the left for CMF. Therefore the idea of using a simulated template was refined and an approach based on a simple circular template with accurate radius estimation was investigated. Since the AMPOF produced better results it was inferred that a high frequency enhancement would work better. Thus we resorted to comparing the edges of the image spots to those of a circular beam with no delay.
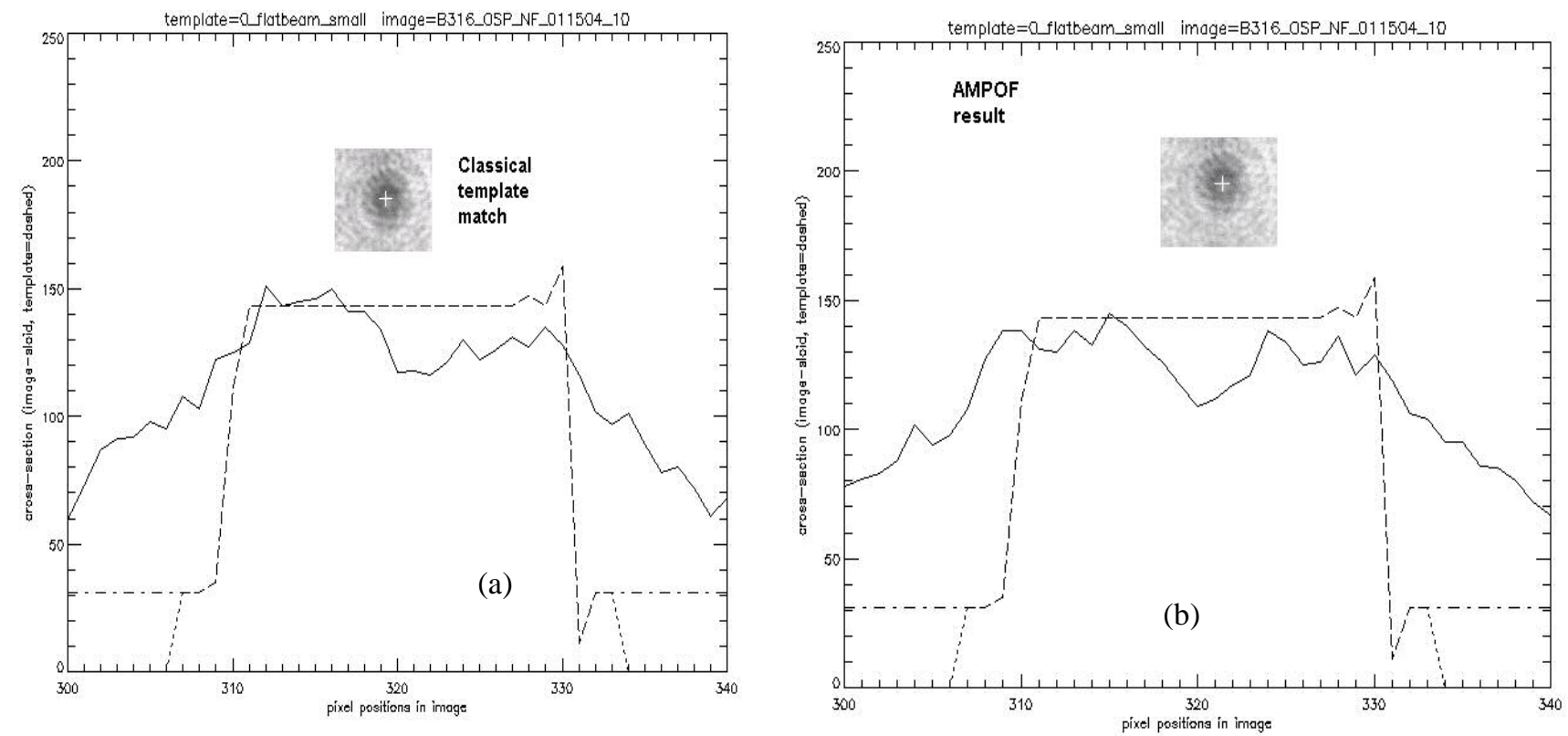

Fig. 8: The classical matched filter output (b) AMPOF based output

The most challenging part of this work was finding the right template size. This was achieved by correlating an attribute of the image spot with the radius of the edges. The pedestal area was defined as the number of pixels within a certain percentage (e.g., 80\%) of the peak pixel intensity value. It should be noted that the image was normalized and inverted, which means the dark regions of the spots in Fig. 5 became the brightest. A plot of the pedestal area versus the optimum radius is shown in Fig. 9. This plot serves as training data for determining the region boundaries. Note from Fig. 9 that when the pedestal area is between 500 and 700 pixels the true radius is fewer than 10 . When it is under 1000 in some cases the radius could be as low as 14 and as high as 20 pixels. 


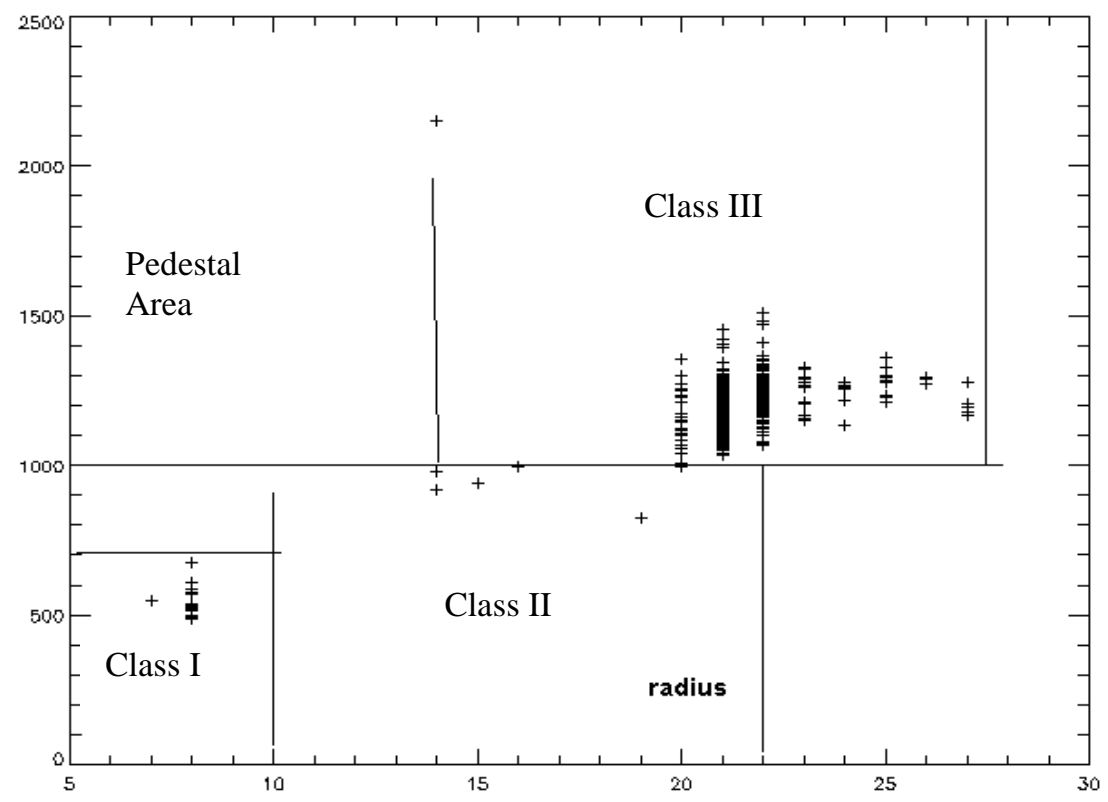

Fig. 9: The plot of the pedestal size versus final selected radius

One way this relationship could be captured is shown in Fig. 10. Note that the pedestal area does not translate into an exact radius estimate but provides a range of radii over which a search is conducted to find the best match. In other words, the correlation over this range of radii is maximized for each image. In Fig. 10 the $r_{1}$ and $r_{2}$ represent the minimum (minRad) and maximum radii (maxRad). Note that when the pedestal is greater than 1000 , we find that a spot producing a pedestal of 2200 has a radius of only 14. In order to include this case in the class III range as shown in Fig. 9, the lower limit of the minRad starts from 14. A typical plot of the search of the radius corresponding to the maximum correlation is shown in Fig. 11.

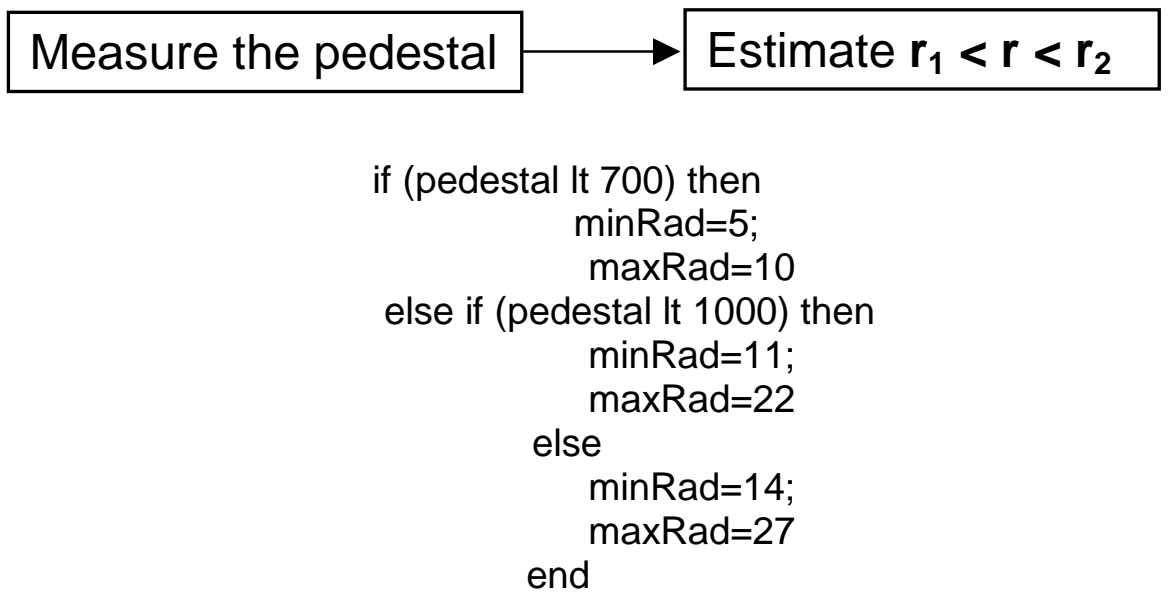

Fig. 10: A classification scheme based in the plot of Fig. 9

Based on the classification algorithm described in Fig. 10, the automated template was generated and then applied to the beam with the expanded center spot as noted in Fig. 5. The template was applied to a set of 30 beam images taken in succession. 


\section{Correlation vs radius}

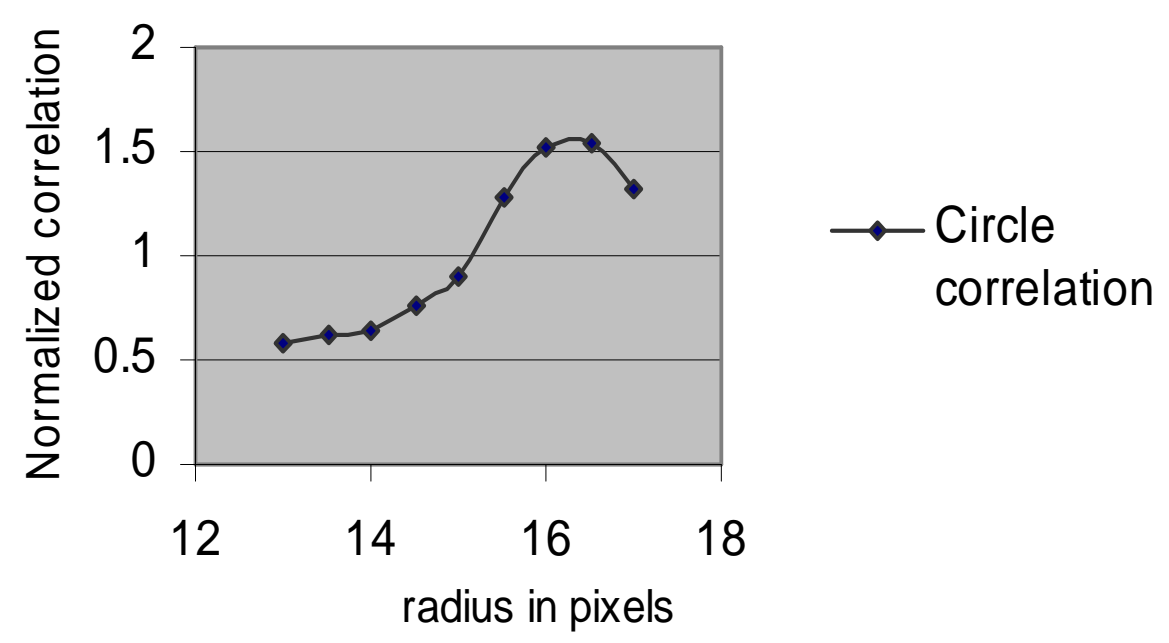

Fig. 11: Results of the search for the optimum radius: between 16 and 17

The resulting beam positions are depicted in a scatter plot shown in Fig. 12. The 3-sigma value was found to be bounded by 0.5 pixels. It should be noted that the pedestal area was affected by beam noise, illumination gradient, and contrast. These factors might change the pedestal area value and lead to a misclassification. A broad range of overlapping radii over which the search was conducted averted such misclassification. If beam quality were better, then the range of radii over which the search was conducted could be lowered. The technique was applied to all beams with different defocus and the output from the algorithm on four sample beams is shown for three types of defocus in Fig. 13.

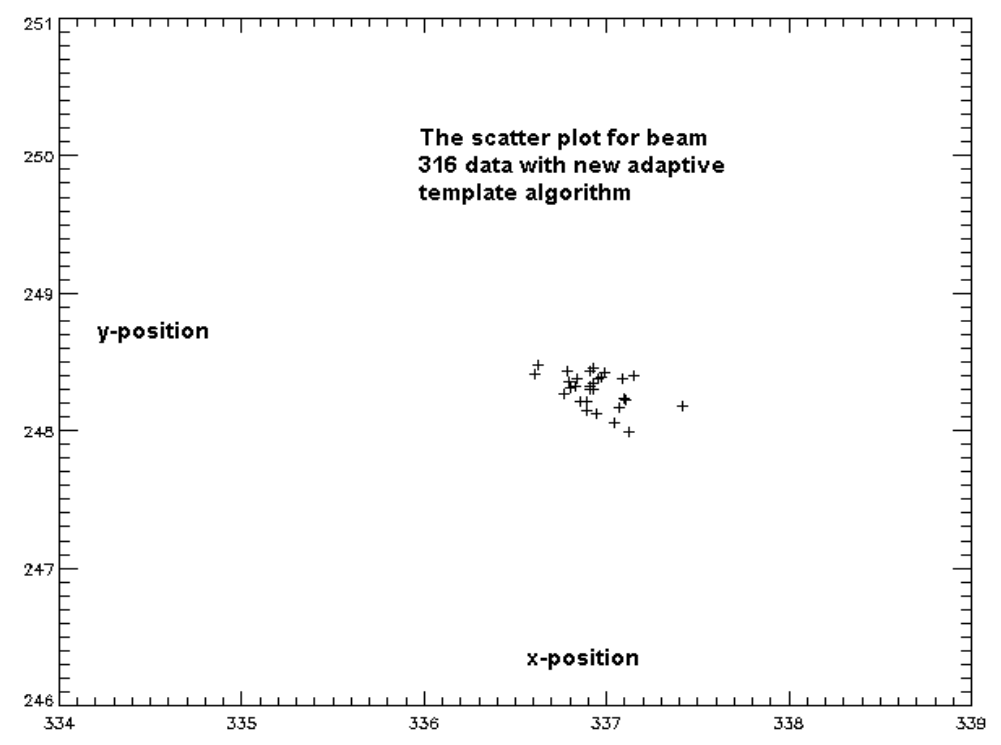

Fig. 12: Scatter plot of a specific beam with automated template generation based on classification 

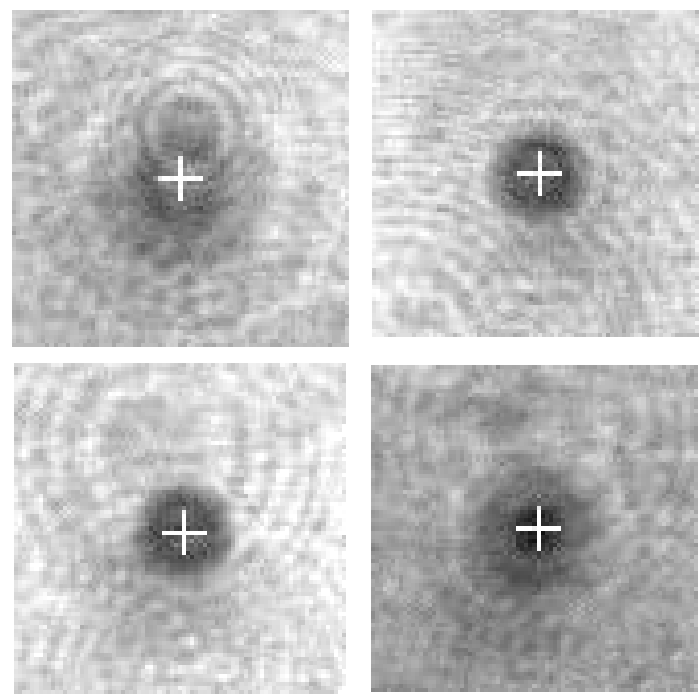

Fig. 13: Results from auto-template with 4 different beams of 2 different beam defocus

\section{CONCLUSIONS}

This paper describes a systematic approach to evaluating competing approaches for solving beam position detection. The use of a simulated template works well if the beam radius can be identified accurately. When the beam radius is determined automatically, this information leads to a simple automated search. The result is equivalent to using the beam with no delay as a template against all other beam delays utilizing knowledge of the approximate radius range. This work shows an interesting application of learning or estimating the beam fiducial radius from beam characteristics such as pedestal area. It may be possible to reduce the range of the searches by incorporating more than one type of characteristic measure, which will lead to a more unique mapping.

\section{ACKNOWLEDGEMENT}

The authors acknowledge useful feedback about the paper by Paul Van Arsdall and technical editing by Sharon Cornelious. This work was performed under the auspices of the U.S. Department of Energy by the University of California, Lawrence Livermore Laboratory under contract No. W-7405-Eng-48.

\section{REFERENCES}

1. E. Moses, et al., "The National Ignition Facility: Status and Plans for Laser Fusion and High-Energy-Density Experimental Studies", Fusion Science and Technology, V. 43, p. 420, May 2003.

2. VanderLugt, "Signal Detection by Complex Spatial Filtering," IEEE Trans. Inf. Theory IT-10, 139-145, 1964.

3. J. L. Horner and J. Leger, "Pattern Recognition with Binary Phase-only Filters," Applied Optics, Vol. 24, pp.609$611,1985$.

4. A. A. S. Awwal, M. A. Karim, and S. R. Jahan, "Improved Correlation Discrimination Using an Amplitudemodulated Phase-only Filter," Applied Optics, Vol. 29, pp. 233-236, 1990.

5. M. A. Karim and A. A. S. Awwal, Optical Computing: An Introduction, John Wiley, New York, NY, 1992.

6. K. M. Iftekharuddin, M. A. Karim, and A. A. S. Awwal, "Optimization of Amplitude Modulated Inverse Filter," Mathematical and Computer Modeling, Vol. 24, pp. 103-112, 1996. 